\title{
Características de las Neoplasias Malignas en el Aparato Digestivo, Callao 1991 - 1995
}

\author{
ALEJANDRO BERMÚDEZ', EDUARDO MONGE'. \\ 'Estudiante del Sexro uño de la Facultad de Medicina, 'Profesor del Depantamento de Medicira -U.N.M.S'M. \\ Senvicio de Gastroenterología «Dr. Meilach Burstein P.», Hospital Nacional Daniel Alcides Carrión.
}

\begin{abstract}
RFSUMEN
Oljetivo: Conocer lá distribución y algunas características de las neoplasias malignas del aparato digestivo en una población del Callizo - Perí.

Diseño: Estudio descriptivo, retrospectivo, observacional.

Contexto: Servicio de Gastroenterología del Hospital Daniel A. Carrión, Callao-Perú.

Participantes: Fucron incluidos en el estudio todos los pacientes con diagnóstico de cáncer del aparato digestivo entre Novienibre de 1991 y Marzo de 1995. Se tomaron cono casos todos los pacientes con diagnóstico de neoplasia maligna de aparato digestivo confirmado nediante estudio anatomopatológico, y considerándose positivo el cáncer de páncreas con una eco-tomografia (+) y PCRF sugerente de diagnóstico.

Intervenciones: Se revisaron los informes de endoscopías, colonosigmoidoscopías, laparoscopías, historias clínicas y PCRE, atendidos en el mismo hospital entre Noviembre de 1991 y Marzo de 1994.

Resultados: Se encontró 252 casos de cáncer del aparato digestivo, con $63,1 \%$ en varones. La neoplasia más común fue la de estómago, en $57,3 \%$ de los pacientes, seguida por la de colon y recto en $15,5 \%$ y en tercer lugar hígado en $9,1 \%$. El $12 \%$ de cánceres gástricos se eucontraron en menores de 45 años y solo el $22 \%$ se encontró limitado al antro. El $80 \%$ de los cánceres colorrectules se encontraron en colon descendente, sigmoides y recto. El cáncer de ampolla duodenal es encontrado 1,8 veces más frecuentemente que el de páncreas.

Conclusiones: El cáncer digestivo es más común cn varones, siendo la localización más frecuente en el estómago. $12 \%$ de cánceres gástricos se observan en menores de 45 años. El $80 \%$ de neoplasias de colon son abordalles por la proctosigmoidoscopía, además el cáncer de ampolla es la neoplasia periampular más frecuente.
\end{abstract}

Palabras Clave: Nesplasmas malignos, Oncología nédica, Carcinoma, Sistema digestivo.

\section{CHARACTERISTICS OF MALIGNANT NEOPLASIAS AT GASTROINTESTINAL TRACT. CALLAO 1991-1995 SUMMARY}

Objetive: To find out the distribution and some characteristics of maliguant neoplasias of the gastrointestinal (GI) tract in a population of Callao - Peru.

Design: A descriptive, retrospective and observational study.

Context: GI unit, Daniel Carrión Hospital, Callao, Peru.

Participan(s: Fach patient with an bistologically documented GI tract cancer, between Novenber 1991 and March 1995, was included in the study. Pancreatic carcinomas were also considered with a positive CAT scan and/or ERCP study.

Intervention: Clinical records, upper GI endoscopic, colonoscopic, laparoscopic and ERCP reports of procedures doue in the study period, were reviewed.

Results: 252 GI tract cancers, were included. $63.1 \%$ were males. The most conmon location was in stomach $(57.3 \%)$, colon and rectum $(\mathbf{1 5 . 5 \%})$ and hepatic carcinomas $(9.9 \%)$. Gastric carcinoma Has found in persons aged 45 or younger in $12 \%$ of cases. Only $22 \%$ of gastric cancers were located on the antrum. $80 \%$ of colorectal cancers were located on the left hemi-colon and rectum. Ampullocarcinoma was 1.8 tinics more frequent than pancreatic callecer.

Conclusions: GI tract cancer was more frequent in the male population. The most conmon location is stomacl. $12 \%$ of the total were found in young patients. $80 \%$ of colon carcinonlas are approachable by the sigmoidoscope. Ampullocarcinoma is more frecuent than pancreatic cancer.

Keywords: Malignant neoplasms, Medical oncology, Carcinoma, Digestive system

Correspondencia: 


\section{INTRODUCCION}

Los tumores malignos son uma patología frecuente en nuestro medio, siendo una causa muy común de defunciones en el mundo. De ellos, el tumor maligno del trácto digestivo, ocupa mundialmente uno de los primeros lugares. (1) Por tanto, conocer sus características, disuibución y relaciones con la edad y el sexo, contribuyen al conocimiento de esta patología en nuestro medio, para porter mejorar la detección precoz y de esta manera ampliar las posibilidades de recuperación, mejorando la calidad de vida de los pacientes con el tratamiento oportuno.

La causa de muerte más frecuente entre los adultos en el Perú es la enfermedad cardiovascular seguida por la neoplasia maligna. De esta última, las neoplasias de la vía digestiva ocupan el primer lugar $\left({ }^{2}\right)$, siendo éslo similar a lo que ocurre en otras partes del mundo ("). Recienlemente, Nago $\left({ }^{4}\right)$ ha publicado una revisión de la frecuencia de las diferentes neoplasias del aparato digestivo en nuestro medio, estos datos son extraidos del Ministerio de Salud, que aun asumiendo un sub-registro significativo, es definitivamente uno de los indicadores más valiosos existentes en el Purú.

Este estudio está dirigido a conocer cual es la realidad de los tumores malignos digestivos en un centro de detección precoz de cáncer como lo es el Servicio de Gastroenterología «Meilach Burstein Pait» del Hospital Nacional Daniel Alcides Carrión del Callao-Perú.

En el presente trabajo describiremos cual ha sido la distribución de las neoplasias malignas del tracto gastrointestinal, en el H.N.D.A.C. durante el período noviembre 1991 -marzo 1995, además de analizar localización, tipos histológicos, edad y sexo en los pacientes afectados con esta palología.

\section{MATERIAL, Y METODOS}

E] presente es un estudio descriptivo, retrospectivo y observacional. Se revisaron las historias clínicas, los reportes endoscópicos, colonoscópicos, pancrealo-colangjográficos y laparoscópicos de los pacientes atendidos en nuestro servicio entre noviembre de $1991 \mathrm{y}$ marzo de 1995; incluyendose todo aquel paciente con neoplasia maligna del aparato digestivo. Se consideró como caso de estudio a todo aquel que tuyiese contirmación bistológica en el caso de esófago, estómago, colon, hígado y ampolla; considerándose como positivo el diagnóstico de cáncer de páncreas con una eco-tomografía (+) y PCRE sugerente del diagnóstico.

Para cada paciente se consignó edad, sexo, localización del tumor y fecha en que se hizo el diagnóstico.

\section{RESULTADOS}

Se estudió 252 casos de neoplasia maligna del aparato digestivo, 159 casos $(63,1 \%)$ fueron hallados en hombres y 93 $(36,9 \%)$ en mujeres. La edad promedio general encontrada fue de 59 años, siendo la menor edad 19 años y la mayor 94.

La distribución general del tumor maligno fue: 11 casos $(4,3 \%)$ en esófago, $145(57,5 \%)$ en estómago, 5 casos $(1,9 \%)$ en duodeno, colon y recto en 39 casos $(15,4 \%)$, en hígado 23 casos $(9,1 \%)$, páncreas con 10 casos $(3,9 \%)$, en ampolla 18 casos $(7,1 \%)$ y un solo caso en vesícula biliar (Tabla $N^{\circ} 1$ ).

Tabla 1.- Distribución de Casos por Ubicación del Cáncer

\begin{tabular}{lrrrrr}
\hline Localización & $\mathrm{n}$ & Masculino & Femenino & \% & Edad $(\overline{\mathrm{x}})$ \\
\hline Esófago & 11 & 7 & 4 & 4,36 & 68 \\
Estómago & 145 & 92 & 53 & 57,53 & 60 \\
Duodeno & 5 & 4 & 1 & 1,98 & 62 \\
Colon y Recto & 39 & 23 & 16 & 15,47 & 61 \\
Hígado & 23 & 13 & 10 & 9,12 & 57 \\
Ves. Biliar & 1 & 1 & 0 & 0,39 & 62 \\
Ampolla & 18 & 13 & 5 & 7,14 & 48 \\
Total & 252 & 159 & 93 & 100,00 & 59 \\
\hline
\end{tabular}

En lo referente a las características de la neoplasia maligna, encontramos que del total de casos en esófago, $63.6 \%$ se ubicaron en el tercio inferior, el $81,8 \%$ ( 9 casos) fueron carcinomas epidermoides (Tabla $N^{\circ} 2$ ), al mismo liempo la edad promedio de presentaciớn fue de 68 años.

Tabla 2.- Características del Cáncer de Esófago Localización y Tipo Histológico

\begin{tabular}{lcccc}
\hline & \multicolumn{3}{c}{ Localización (\%) } & \\
\cline { 2 - 4 } Tipo & \multicolumn{1}{c}{ Tercio Sup. Tercio Med. Tercio Inf. } & Total \\
\hline & & & & \\
Epidermoide & $0(0,0)$ & $4(44,4)$ & $5(55,6)$ & 9 \\
Adenocarcinoma & $0(0,0)$ & $0(0,0)$ & $2(100,0)$ & 2 \\
Total & 0 & 4 & 7 & 11 \\
\hline
\end{tabular}

En el análisis del cáncer de estómago se observó compromiso limitado al antro en 32 casos $(22,1 \%)$, en cuerpo y/o cuerpo y antro 66 casos $(45,5 \%)$ y en fondo y/o fondo, cuerpo y antro en 41 casos $(28,2 \%)$. La bistopatología mas frecuente fue el carcinoma indiferenciado con 74 casos $(71 \%)$, frente al diferenciado, $61(42 \%)$ casos (Tabla $N^{\circ} 3$ ). En el resto de casos no se tuvo precisión de la patologfa. La edad promedio de presentación fue de 60 años, observándose que 17 casos $(11,7 \%)$ se presentaron en menores de 40 años y 75 casos $(51,7 \%)$ en mayores de 75 años (Tabla $\mathrm{N}^{\circ} 4$ ). A su vez la.presentación de cáncer en fondo gástrico es similar tanto en menores de 40 años $(29,4 \%$ ) y mayores de 65 años (32\%), 
Tabla 3.- Características del Cáncer de Estómago Localización y Tipo Histológico

\begin{tabular}{lccccc}
\hline & \multicolumn{4}{c}{ Localización } & \\
\cline { 2 - 5 } Tipo & Fondo & Cuerpo Antro & No Det. & Total \\
\hline Indiferenciado & 23 & 33 & 15 & 3 & 74 \\
Diferenciado & 13 & 30 & 16 & 2 & 61 \\
No Determinado & 5 & 3 & 1 & 1 & 10 \\
Total & 41 & 66 & 32 & 6 & 145 \\
\hline
\end{tabular}

Tabla 4.- Características del Cáncer de Estómago. Grupo Etáreo y Sexo

\begin{tabular}{|c|c|c|c|}
\hline \multirow[b]{2}{*}{ Grupo } & \multicolumn{2}{|c|}{ Sexo } & \multirow[b]{2}{*}{ Total } \\
\hline & Masculino & Femenino & \\
\hline Menor de 40 años & 8 & 9 & 17 \\
\hline Mayor de 65 años & 56 & 19 & 75 \\
\hline Total & 64 & 28 & 92 \\
\hline
\end{tabular}

El duodeno presentó poca frecuencia de neoplasias malignas en nuestra muestra, con preferencia por el sexo masculino $(80 \%)$ y una edad promedio de 62 años.

La neoplasia de ámpolla se presentó preferentemente en el sexo masculino (72\%) y se observó igualmente en pacientes menores de 40 años así como en mayores de 65 años. La edad́ promedio fue de 48 años.

En el colon y recto, observamos que el segundo presenta el mayor número de casos $(54,3 \%)$ seguido por colon sigmoides y ascendente, 5 casos cad'a uno $(14,3 \%)$, luego colon descendente $(11,4 \%)$ y finalmente ciego y transverso con un caso cada uno $(2,8 \%)$. La presencia en sexo masculino fue de 21 casos $(60 \%)$ y en el femenino (40\%). La edad promedio de presentación fue de 6I años. Se presentó en menores de 40 años, 4 casos todos en localización distal (descendente, sigmoides y recto), frente a los mayores de 65 años, donde 15 casos fueron de localización distal a predominio de recto con 11 casos y 3 en colon proximal (Tabla $\mathrm{N}^{\circ} 5$ ).

En hígado encontramos 23 neoplasias malignas de las cuales $14(60,8 \%)$ fueron primarias y 9 metastásicas $(39,2 \%)$ siendo la primaria más común en varones $(71 \%$ ) con una edad promedio de 57 años (Tabla $N^{\circ} 6$ ).
Tabla 5. Características del Cáncer de Colon y Recto. Localización y Sexo.

\begin{tabular}{lccc}
\hline & \multicolumn{3}{c}{ Sexo } \\
\cline { 2 - 3 } Localización & Masculino & Fernenino & Total \\
\hline Ciego & 1 & 0 & $1(2,85 \%)$ \\
Ascendente & 4 & 1 & $5(14,28 \%)$ \\
Transverso & 1 & 0 & $1(2,85 \%)$ \\
Descendente & 3 & 1 & $4(11,4 \%)$ \\
Sigmoides & 2 & 3 & $5(14,28 \%)$ \\
Recto & 10 & 9 & $19(54,28 \%)$ \\
Total & $21(60 \%)$ & $14(40 \%)$ & $35(100,0 \%)$ \\
\hline
\end{tabular}

Tabla 6.- Clasificación de la Neoplasia Hepática según su Origen

\begin{tabular}{lccc}
\hline Tipo & Masculino & Femenino & Total \\
\hline Primario & 10 & 4 & 14 \\
Secundario & 4 & 5 & 9 \\
Total & 14 & 9 & 23 \\
\hline
\end{tabular}

\section{DISCUSION}

En el mundo, la neoplasia digestiva, según múltipless series, se ubica dentro de las más importantes $\left({ }^{3.6}\right)$, encabezando la lista el tumor maligno de estómago $\left({ }^{2.6}\right)$, es por ello, la importancia de una prevención secundaria para evitar la muerte por este tipo de neoplasia en nuestro medio $\left({ }^{4}\right)$.

Para poder estructurar un plan de detección de cáncer de aparato digestivo, es necesario conocer la distribución de esta patología, de esta manera podremos optimizar los escasos recursos con los que contamos para el diagnóstico de los casos y así aumentar la esperanza de vida de nuestra población con el tratamiento oportuno.

Es así que tomamos como referencia la casuística del Servicio de Gastroenterología del HNDAC-Callao para determinar esta distribución en nuestro medio.

En poco más de tres años en este servicio se detectó 252 casos de neoplasia maligna de aparato digestivo. De ellos, se aprecia un mayor número de pacientes de sexo masculino, cabiendo resaltar que la atención en este servicio es similar tanto para población masculina como para femenina.

Entre los resultados encontrado observamos un gran predominio de cáncer gástrico, lo cual es semejante a lo reportado 
en otras series nacionales ( $\left.{ }^{4}\right)$, así como en Japón, Chile, Costa Rica, etc. $\left({ }^{2}\right)$, siendo éste más del $50 \%$ de la totalidad de los cánceres del aparato digestivo.

En lo referente a otras partes del aparato digestivo nuestra distribución es semejante a la de Nago ( $\left.{ }^{(}\right)$, es decir ocupando el segundo lugar colon y recto, luego páncreas y esófago. La diferencia radica en la desproporcionadamente alta incidencia de cáncer de ampolla, que puede estar relacionada con los nuevos métodos diagnósticos como la PCRE, además, en este lapso de tiempo el servicio fue el único que contó (a nivel del Ministerio de Salud) con este tipo de equipo diagnóstico. A nivel de esófago la edad promedio fue de 68 años, es decir 10 años más que la edad promedio total, lo cual respalda la idea que el cáncer de esófago es más frecuente en ancianos ( ${ }^{7}$ ). Al mismo tiempo, el carcinoma epidermoide se presenta en un porcentaje elevado, semejante a las series americanas ( ); a la vez, la mayor incidencia en hombres sobre mujeres es semejante a otros estudios ( ${ }^{3}$. $\left.{ }^{7}\right)$. Se debe resaltar que la ubicación en tercio medio es elevada, similar a otros estudiós.

A nivel de estómago, hallamos que la neoplasia es mas coinún en hombres que en mujeres, como está descrito $\left(^{3}\right)$, en lo referente a edad es de recalcar que $12 \%$ de nuestra población son inenores de 45 años, ésto es importante porque a diferencia dc lo que recomiendan autores europeos $\left({ }^{8}\right)$, en nuestro medio si es necesario descartar el cáncer gástrico en gente joven.

Es importante reconocer que el carcinoma indiferenciado es más frecuente que el diferenciado, como hacen referencia algunos estudios del mismo servicio (9.10, Arrús y cols., comunicación personal).

Desde el punto de vista de manejo del cáncer gástrico, es importante determinar la localización de la neoplasia: ballando $22 \%$ limitado al antro, que en caso de tratamiento estarían sujetos a gastrectomía parcial $\left({ }^{11}\right)$, mientras que compromisos mas altos (cuerpo y fondo) están sujetos a gastrectomía total.

En neoplasia maligna de colon debemos recalcar que el $80 \%$ de nuestros casos esián localizados en colon distal (descendente, sigmoides y recto). Su importancia radica en la bondad del examen, pues mediante el estudio simple con proctosigmoidoscopía flexible, estamos evaluando un importante porcentaje de neoplasias en esta región. Es relevante manifestar que el $50 \%$ de neaplasia de colon proximal (ciego, ascendente y transverso) se presenta en mayores de 65 años $\left({ }^{12}\right)$, y ninguno en menores de 45 años, tal como lo reportado por Fleishner y col $\left({ }^{13}\right)$.

En lo referido a carcinoma periampular, en diferentes series (3.4.14) eacontramos mayor frecuencia de cáncer de páncreas que de neoplasia maligna de ampolla; en nuestra serie por el contrario esta relación se invierte. Esto podría ser debido a la introducción de métodos diagnósticos como la PCRE, en nues- tro servicio con lo que lo convirtió en centro de referencia para este tipo de estudio.

En nuestra serie en lo referente a cáncer hepático, el número de casos de tumor primario y secundario es semejante.

\section{CONCLUSIONES}

1. El cáncer digestivo es 1,7 veces más frecuente en hombres que en mujeres, y esta relación es semejante en todas las ubicaciones.

2. El cáncer gástrico, es la neoplasia maligna del aparato digestivo más frecuente, con un porcentaje del 57,3\%.

3. El $12 \%$ de cáncer gástrico se produce en personas menores de 45 años de edad.

4. El $80 \%$ de neoplasias malignas de colon, están al alcance de la proctosigmoidoscopía flexible.

5. El cáncer de ampolla, es la neoplasia maligna periampular más frecuente.

\section{BIBLIOGRAFIA}

1) Lippnan SM; Spitz M; Trizna Z. Epidemiology, bialogy, and chemoprovention of aerodigestive cancer. Cancer 1994; 74(9 Supp 1): $2719-25$.

2) Silver E, Boring C, Squire T. Cancer Statistics, 1990. Ca - A Cancer Journal of Clinicians 1990; 40: 9-26.

3) Yamada T, Alpers D, Owyang C,et al. Texibook of Gastroenterology $1^{\circ}$ Edic. I B Lippincoll Company Philadelphia 1991.

4) Nago A. Epidemiología de las Enfermedades Digestivas Gastroenterología III 1994 5-15

5) Faggiano F; Zantti R; Costa G. Cancer risk and sucial inequalities in Italy. J Epideniol Conunity Health 1994; 48(5): 447-52.

6) Mc Credle M; Coates M; Grulich A. Cancer incidence in migrants to New Soutb Wales 1972-91. Caucer Causes Coptrol 1994; 5: 414-21.

7) Carl B, Wallach and Robert C, Jacobson. Gastrointestinal Cancer in the Elderly. Gastroenterology Clinics of Nortb America. June 1990;19:- 273.292.

8) Williams B, Ellinghan JHM, Luckas $M$, et al. Do young patients with Dyspepsia Need Inyestigation. Ladcet Decerober 1988; 2:13491351.

9) Beteta O, Lozano R, Monge E. et al Change in the histology type of gastric carcinoma. Am. J. Gastroenterology 1993;88:787-7.

10) Szende B; Kendrey G; Lapis K; Lee PN; Roe F.J. Accuracy of admission an pre-autopsy cliuical diagnoses in the light of autopsy findings: a study conducted in Budapest. Hum Exp Toxicol 1994; 13(10): $671-80$

11) Romero Torres Raúl. Tratado de Cirugía. México D.F. Nueva Editorial Interamericana 1 ed. Vol 2 1431-48. 1985.

12) Lin M; Налаj J; Wan Y; Du $X_{\text {; }}$ Gui L. Differences in colorectal cancer between China and Japan: a clinical an histological study of two groups of cases. Eur J Cancer Prev 1995; 4(2): 195-9.

13) Fleshner P, Slater G, Aufses A. Age and Sex distribution of patient with colo-rectal cancer. Dis colon rect 1989, 32: $107-11$.

14) Nakaizumi A; Uehara H; Iissi H; Tatsuta M; Kitamura T; Kuruoda C. Endoscopic ultrasonogrphy in diagnosis and staging of pancreatic cancer. Dig Dis Sci 1995; 40(3): 696-700. 